\title{
ON THE STONE-ČECH COMPACTIFICATION OF THE SPACE OF CLOSED SETS
}

\author{
BY \\ JOHN GINSBURG( ${ }^{1}$ )
}

\begin{abstract}
For a topological space $X$, we denote by $2^{X}$ the space of closed subsets of $X$ with the finite topology. If $X$ is normal and $T_{1}$, the map $F$ $\rightarrow \mathrm{cl}_{\beta X} F$ is an embedding of $2^{X}$ onto a dense subspace of $2^{\beta X}$, and, in this way, we regard $2^{\beta X}$ as a compactification of $2^{X}$. This paper is motivated by the following question. When can $2^{\beta X}$ be identified as the Stone-Čech compactification of $2^{X}$ ? In [11], J. Keesling states that $\beta\left(2^{X}\right)=2^{\beta X}$ implies $2^{X}$ is pseudocompact. We give a proof of this result and establish the following partial converse. If $2^{X} \times 2^{X}$ is pseudocompact, then $\beta\left(2^{X}\right)=2^{\beta X}$. A corollary of this theorem is that $\beta\left(2^{X}\right)=2^{\beta X}$ when $X$ is $\aleph_{0}$-bounded.
\end{abstract}

1. Introduction and preliminaries. Our topological notation and terminology follows [3], where the reader may find any required background material on compactifications. In particular, we denote the Stone-Čech compactification of a completely regular Hausdorff space $X$ by $\beta X$. For a space $X, C(X)$ denotes the ring of continuous real-valued functions on $X$, and $C^{*}(X)$ its subring of bounded functions. The set of zeros of $f \in C(X)$, that is, $f^{-1}(\{0\})$, will be denoted by $Z(f)$. The family of all such zero sets of functions in $C(X)$ is denoted by $Z(X)$.

The subject of hyperspaces has been studied by several authors from several points of view, notably E. Michael [14], J. Keesling [8] , [9] , [10] , [11], P. Zenor [17], and K. Morita [15]. For the fundamental properties of hyperspaces, our basic references are [12] and [14]. We are concerned here with compactifications of hyperspaces. In this section, we recall the definition of hyperspaces, and state several basic facts about hyperspaces which are relevant to the theme of this paper.

Definition 1.1. Let $X$ be a topological space. Let $2^{X}$ denote the set of all nonempty closed subsets of $X$. For a subset $A$ of $X$, we let $2^{A}=\left\{F \in 2^{X}\right.$ : $F \subseteq A$ \}. We generate a topology on $2^{X}$ by taking all sets of the form $2^{G}$ and all

Received by the editors December 5, 1974.

AMS (MOS) subject classifications (1970). Primary 54B20, 54D35; Secondary 54C45.

Key words and phrases. Hy perspace, Stone-Čech compactification, pseudocompactness, topological semilattice.

(1) This research was partially supported by a fellowship from the National Research Council of Canada. 
sets of the form $2^{X}-2^{X-G}$, for $G$ open in $X$, as a subbasis. This topology on $2^{X}$ is known as the finite topology and $2^{X}$, endowed with this topology, is called the hyperspace of $X$.

Following [12], we make the following notational convention. For subsets $A_{0}, A_{1}, \ldots, A_{n}$ of $X$, we let $B\left(A_{0} ; A_{1}, \ldots, A_{n}\right)=2^{A} 0 \cap \bigcap_{i=1}^{n}\left(2^{X}-2^{X-A_{i}}\right)$ $=\left\{F \in 2^{X}: F \subseteq A_{0}\right.$ and $F \cap A_{i} \neq \varnothing$ for all $\left.i=1,2, \ldots, n\right\}$. Using this notation we see that the sets $B\left(G_{0} ; G_{1}, \ldots, G_{n}\right)$, where $G_{0}, G_{1}, \ldots, G_{n}$ are open in $X$ and $\bigcup_{i=1}^{n} G_{i} \subseteq G_{0}$, form a basis for $2^{X}$.

We now state several basic facts about hyperspaces which we will need in the course of our discussion.

(i) $2^{X}$ is compact Hausdorff $\Longleftrightarrow X$ is compact Hausdorff [14].

(ii) $2^{X}$ is completely regular and Hausdorff $\Longleftrightarrow X$ is normal and $T_{1}$ [14].

(iii) If $X$ is normal and $T_{1}$, the natural mapping $i: 2^{X} \rightarrow 2^{\beta X}$ defined by $i(F)=\mathrm{cl}_{\beta X} F$ is an embedding of $2^{X}$ onto a dense subspace of $2^{\beta X}$ [8].

(iv) The union operation, $(A, B) \rightarrow A \cup B$, from $2^{X} \times 2^{X}$ into $2^{X}$, is continuous [12].

(v) If $X$ is Hausdorff, the set of singleton sets in $2^{X}$ is a closed subset of $2^{X}$ homeomorphic to $X$ [14].

(vi) Let $f$ be a bounded, real-valued function on $X$. We define real-valued functions $f^{s}$ and $f^{i}$ on $2^{X}$ by

$$
f^{s}(F)=\sup \{f(x): x \in F\} \quad \text { and } \quad f^{i}(F)=\inf \{f(x): x \in F\} .
$$

Then if $f$ is continuous, so are $f^{i}$ and $f^{s}$. We have for $f \geqslant 0, Z\left(f^{s}\right)=2^{Z(f)}$ and, if $X$ is countably compact, $Z\left(f^{i}\right)=B(X ; Z(f))$. Identifying $X$ with the singletons in $2^{X}$, we get that, for Hausdorff $X, X$ is $C^{*}$-embedded in $2^{X}$ [14].

(vii) If $X$ is normal and $T_{1}$, the sets of the form $B\left(X ; Z_{0}\right) \cup 2^{Z_{1}} \cup \ldots$ $\cup 2^{Z}{ }_{n}$, where $Z_{0}, Z_{1}, \ldots, Z_{n} \in Z(X)$, form a base for the closed sets in $2^{X}$.

Since in this paper we are concerned with compactifications of hyperspaces, we are forced to restrict our discussion to normal $T_{1}$ spaces $X$, for, by (ii), these are precisely those $X$ whose hyperspaces have compactifications. If $X$ is normal and $T_{1}$, we see, by (i) and (iii), that $2^{\beta X}$ is a compactification of $2^{X}$. This paper is motivated by the following question. When can $2^{\beta X}$ be identified as the StoneČech compactification of $2^{X}$ ? In $\S 2$ we give a necessary condition for this to occur, a result obtained by the author, and independently by $\mathrm{J}$. Keesling, who announced it in [11], namely: if $\beta\left(2^{X}\right)=2^{\beta X}$, then $2^{X}$ is pseudocompact. $\S 3$ is devoted to the converse of this statement. We obtain the following partial converse: if $2^{X} \times 2^{X}$ is pseudocompact, then $\beta\left(2^{X}\right)=2^{\beta X}$. A corollary of some interest is the following. If $X$ is $\aleph_{0}$-bounded, then $\beta\left(2^{X}\right)=2^{\beta X}$.

We remind the reader that throughout this paper we are assuming that $X$ is normal and $T_{1}$, and this assumption will often be used without explicit mention. 
2. A necessary condition for $\beta\left(2^{X}\right)=2^{\beta X}$. Our first result gives a necessary condition for $\beta\left(2^{X}\right)=2^{\beta X}$. This theorem was also established independently by J. Keesling, who announced it without proof in [11].

THEOREM 2.1. If $\beta\left(2^{X}\right)=2^{\beta X}$, then $2^{X}$ is pseudocompact.

Proof. Before proceeding with the argument, let us examine the meaning of the equality $\beta\left(2^{X}\right)=2^{\beta X}$. Let $i: 2^{X} \rightarrow 2^{\beta X}$ be the canonical embedding of $2^{X}$ onto a dense subspace of $2^{\beta X}$. Now to say that $2^{\beta X}$ is $\beta\left(2^{X}\right)$ is exactly the statement that $i\left(2^{X}\right)$ is $C^{*}$ embedded in $2^{\beta X}$. So assume that $\beta\left(2^{X}\right)=2^{\beta X}$. We will first show that $X$ must be pseudocompact. For the sake of contradiction, suppose $X$ is not pseudocompact. Then there is a sequence $\left(G_{n}: n \in N\right)$ of nonempty open subsets of $X$ with the property that $\mathrm{cl}_{X} G_{n+1} \subseteq G_{n}$ for all $n$, and such that $\bigcap_{n \in N} G_{n}=\varnothing$. We may assume that $\mathrm{cl}_{X} G_{n+1} \subsetneq G_{n}$ for each $n$. For each $n$, we set $F_{n}=\mathrm{cl}_{X} G_{n+1}$, and we define the following sequence of open sets in $2^{X}$. We let $G_{n}=B\left(G_{n} ; X-F_{n+1}\right)=2^{G} n \cap\left(2^{X}-2^{F}{ }^{n+1}\right)$ for each $n$. Then $F_{n} \in G_{n}$ for each $n$. We claim that $\left(G_{n}: n \in N\right)$ is locally finite. For let $A \in 2^{X}$. Let $p \in A$. Since $\bigcap_{n \in N} F_{n}=\varnothing$, there is an integer $k$ such that $p \notin$ $F_{k}$. But then $B\left(X ; X-F_{k}\right)$ is a neighborhood of $A$ in $2^{X}$ meeting only finitely many $G_{n}$. Therefore $\left(G_{n}: n \in N\right)$ is locally finite. Now, let $D=\left\{F_{n}: n \in N\right\}$. Since $G_{n} \cap D=\left\{F_{n}\right\}, D$ is a discrete subset of $2^{X}$. So we may find a sequence $\left(H_{n}: n \in N\right)$ of pairwise disjoint open subsets of $2^{X}$ such that $F_{n} \in H_{n}$ for each $n$. Let $U_{n}=H_{n} \cap G_{n}$ for each $n$. Then $\left(U_{n}: n \in N\right)$ is a locally finite sequence of pairwise disjoint open sets in $2^{X}$, and $F_{n} \in U_{n}$ for each $n$. It follows that $D$ is $C^{*}$-embedded in $2^{X}$. Applying the homeomorphism $i$, we see that $\left\{i\left(F_{n}\right): n \in\right.$ $N$ \} is $C^{*}$-embedded in $i\left(2^{X}\right)$. Since $\beta\left(2^{X}\right)=2^{\beta X}, i\left(2^{X}\right)$ is $C^{*}$-embedded in $2^{\beta X}$. Thus, $\left\{i\left(F_{n}\right): n \in N\right\}$ is $C^{*}$-embedded in $2^{\beta X}$. But since the $F_{n}$ 's descend, it is easy to see that, in $2^{\beta X}, \lim _{n \rightarrow \infty} i\left(F_{n}\right)=\bigcap_{n \in N} i\left(F_{n}\right)$. Letting $L=$ $\bigcap_{n \in N} i\left(F_{n}\right)$, we see that no function on $\left\{i\left(F_{n}\right): n \in N\right\}$ which is 0 for infinitely many $i\left(F_{n}\right)$ and 1 for infinitely many $i\left(F_{n}\right)$ can be extended continuously to $L$. But this is a contradiction, as $\left\{i\left(F_{n}\right): n \in N\right\}$ is $C^{*}$-embedded in $2^{\beta X}$. So we see that indeed $X$ is pseudocompact. Now we show that $2^{X}$ is pseudocompact. To do this, we use the following familiar characterization of pseudocompactness: A completely regular space $Y$ is pseudocompact if, and only if, every nonempty $G_{\delta}$ set in $\beta Y$ meets $Y$. In light of this characterization, to show $2^{X}$ is pseudocompact, we need only show that every nonempty $G_{\delta}$ in $2^{\beta X}$ intersects $i\left(2^{X}\right)$. Thus let $S$ be a nonempty $G_{\delta}$ in $2^{\beta X}$. Find a sequence $\left(G_{n}: n \in N\right)$ of open subsets of $2^{\beta X}$ such that $S=\bigcap_{n \in N} G_{n}$. Let $A \in S$. Then, for each $n$, we can find open sets $G_{n, 0}, G_{n, 1}, \ldots, G_{n, K_{n}}$ in $\beta X$ so that $A \in B\left(G_{n, 0} ; G_{n, 1}, \ldots, G_{n, K_{n}}\right)$ $\subseteq G_{n}$. For each $n$, let $H_{n, 0}$ be open in $\beta X$ such that $A \subseteq H_{n, 0} \subseteq \mathrm{cl}_{\beta X} H_{n, 0} \subseteq G_{n, 0}$. For all $n$, and for all $j \in\left\{1,2, \ldots, K_{n}\right\}$, let $H_{n, j}=G_{n, j} \cap H_{n, 0}$. Then 
$A \in \bigcap_{n \in N} B\left(H_{n, 0} ; H_{n, 1}, \ldots, H_{n, K_{n}}\right) \subseteq \bigcap_{n \in N} B\left(G_{n, 0} ; G_{n, 1}, \ldots, G_{n, K_{n}}\right) \subseteq S$.

Let $H=\bigcap_{n \in N} H_{n, 0}$, and let $G=\bigcap_{n \in N} G_{n, 0}$. Obviously $\mathrm{cl}_{\beta X} H \subseteq G$. Now, for each $n$ and $j \in\left\{1,2, \ldots, K_{n}\right\}, H_{n, j} \cap H$ is a nonempty $G_{\delta}$ in $\beta X$. By the pseudocompactness of $X$, we can find, for each $n$ and each $j \in\left\{1,2, \ldots, K_{n}\right\}$, a point $x_{n, j}$ in $H_{n, j} \cap H \cap X$. Let $B=\operatorname{cl}_{\beta X}\left\{x_{n, j}: n \in N, j \in\left\{1,2, \ldots, K_{n}\right\}\right\}$. Then $B \in i\left(2^{X}\right)$ and clearly

$$
B \in \bigcap_{n \in N} B\left(G_{n, 0} ; G_{n, 1}, \ldots, G_{n, K_{n}}\right) \subseteq S .
$$

Therefore every nonempty $G_{\delta}$ in $2^{\beta X}$ meets $i\left(2^{X}\right)$. Thus $2^{X}$ is pseudocompact.

In the next section, we will establish a partial converse to 2.1. Before doing so, we offer the following two descriptions of the relation $\beta\left(2^{X}\right)=2^{\beta X}$.

THEOREM 2.2. (i) Let A denote the subalgebra of $C^{*}\left(2^{X}\right)$ generated by $\left\{f^{s}: f \in C^{*}(X)\right\}$. Then $\beta\left(2^{X}\right)=2^{\beta X}$ if, and only if, $A$ is uniformly dense in $C^{*}\left(2^{X}\right)$.

(ii) Let $2^{X}$ be pseudocompact. Then $\beta\left(2^{X}\right)=2^{\beta X}$ if, and only if, every zero set in $2^{X}$ is a countable intersection of basic zero sets of the form $B\left(X ; Z_{0}\right) \cup$ $2^{Z_{1}} \cup \cdots \cup 2^{Z_{n}}$, for $Z_{0}, \ldots, Z_{n} \in Z(X)$.

PRoof. (i) Recall from $\S 1$ that $f^{s}$ is defined on $2^{X}$ by $f^{s}(F)=$ $\sup \{f(x): x \in F\}$. We first observe that each $f^{s}$ can be extended continuously to $2^{\beta X}$. This is immediate, since, given $f \in C^{*}(X)$, we can extend $f$ continuously to $f^{\beta} \in C^{*}(\beta X)$, and then $\left(f^{\beta}\right)^{s}$ is a continuous real-valued function on $2^{\beta X}$ whose restriction to $2^{X}$ (recall we are identifying $2^{X}$ as the subspace $i\left(2^{X}\right)$ of $2^{\beta X}$ ) is clearly $f^{s}$. It follows that every function in $A$ can be extended continuously to $2^{\beta X}$, and thus that every function in the uniform closure of $A$ in $C^{*}\left(2^{X}\right)$ may be so extended. (See [6, Proposition 5].) So if $A$ is uniformly dense in $C^{*}\left(2^{X}\right)$, every function in $C^{*}\left(2^{X}\right)$ extends continuously to $2^{\beta X}$. That is, $\beta\left(2^{X}\right)$ $=2^{\beta X}$. Conversely, if $\beta\left(2^{X}\right)=2^{\beta X}$, then $C^{*}\left(2^{X}\right)$ and $C^{*}\left(2^{\beta X}\right)$ are uniformly isomorphic, under the map $g \rightarrow g^{\beta}$, where $g^{\beta}$ represents the Stone extension to $2^{\beta X}$ of $g \in C^{*}\left(2^{X}\right)$. In light of the preceding remarks we see that $\left(f^{s}\right)^{\beta}=\left(f^{\beta}\right)^{s}$, for all $f \in C^{*}(X)$. And so the converse in question becomes equivalent to the following assertion: If $X$ is compact, the subalgebra of $C^{*}\left(2^{X}\right)$ generated by the functions $\left\{f^{s}: f \in C^{*}(X)\right\}$ is uniformly dense in $C^{*}\left(2^{X}\right)$. This assertion follows from the Stone-Weierstrass theorem, since $\left\{f^{s}: f \in C^{*}(X)\right\}$ contains the constant functions on $2^{X}$, and separates points and closed sets, by facts (vi) and (vii) in $\$ 1$.

(ii) We first show that if $X$ is compact every zero-set in $2^{X}$ is a countable intersection of basic sets of the form $B\left(X ; Z_{0}\right) \cup 2^{Z_{1}} \cup \cdots \cup 2^{Z_{n}}$, where $Z_{0}$, $Z_{1}, \ldots, Z_{n} \in Z(X)$. So, let $W$ be a zero-set in $2^{X}$, with $X$ compact. Find open 
sets $\left(G_{n}: n \in N\right)$ in $2^{X}$ so that $W=\bigcap_{n \in N} G_{n}$. Now, by (vii) in $\S 1$, every closed set in $2^{X}$ is an intersection of certain of the basic sets in question. In particular this is true of $W$. So write $W=\bigcap_{i \in I} A_{i}$, where each $A_{i}$ is a basic set of the form $B\left(X ; Z_{0}\right) \cup 2^{Z} \cup \cdots \cup 2^{Z}$. For each $n, W \subseteq G_{n}$, and so, by the compactness of $2^{X}$, there is, for each $n$, a finite subset $I_{n}$ of $I$ such that $W \subseteq$ $\bigcap_{i \in I_{n}} A_{i} \subseteq G_{n}$. Letting $J=\bigcup_{n \in N} I_{n}$, we see that $J$ is countable and $W=$ $\bigcap_{i \in J} A_{i}$. Thus $W$ is a countable intersection as required.

Now, if $\beta\left(2^{X}\right)=2^{\beta X}$, every zero-set in $2^{X}$ is the restriction of a zero-set in $2^{\beta X}$. Since $\beta X$ is compact, the representation in (ii) holds for zero-sets in $2^{\beta X}$, as proved above, and so, by restriction to $2^{X}$, the corresponding representation for zero-sets in $2^{X}$ is also valid. There is one minor detail to be checked here, namely, that if $Z$ is a zero-set in $\beta X$, then $B(\beta X ; Z) \cap 2^{X}=B(X ; Z \cap X)$. This can be verified as follows. Since $2^{X}$ is pseudocompact, so is $X$, as is easily seen. Since $X$ is normal, $X$ is countably compact, and every closed subset of $X$ is countably compact and $C^{*}$-embedded in $X$. So, if $A \in 2^{X}, \mathrm{cl}_{\beta X} A=\beta A$. If $Z$ is a zero-set in $\beta X$ such that $\left(\mathrm{cl}_{\beta X} A\right) \cap Z \neq \varnothing$, then $\left(\operatorname{cl}_{\beta X} A\right) \cap Z$ is a nonempty zero-set in $\beta A$. Since $A$ is pseudocompact, indeed countably compact, it follows that $\left(\mathrm{cl}_{\beta X} A\right) \cap Z$ meets $A$. That is, $Z \cap A=(Z \cap X) \cap A \neq \varnothing$. Therefore $B(\beta X ; Z) \cap 2^{X}=B(X ; Z \cap X)$.

Now, for the converse of (ii). We assume that $2^{X}$ is pseudocompact, and that every zero-set in $2^{X}$ has the indicated representation. Observe that, since the functions $f^{s}$ and $f^{i}$ on $2^{X}$ extend continuously to $2^{\beta X}$, namely to $\left(f^{\beta}\right)^{s}$ and $\left(f^{\beta}\right)^{i}$, every basic zero-set $2^{Z} \cup \cdots \cup 2^{Z} \cup \cup B\left(X, Z_{n+1}\right)$ in $2^{X}$ is the restriction of a zero-set in $2^{\beta X}$. This is clear from statement (vi) in $\S 1$. Now since a countable intersection of zero-sets is again a zero-set, if every zero-set in $2^{X}$ has a countable representation as in (ii), we conclude that every zero-set in $2^{X}$ is the restriction of a zero-set in $2^{\beta X}$. Together with the pseudocompactness of $2^{X}$, this enables us to conclude by $[1,4.4]$ that $2^{X}$ is $C^{*}$-embedded in $2^{\beta X}$, that is, $\beta\left(2^{X}\right)=2^{\beta X}$.

3. The main theorem. It is our aim in this section to establish a partial converse to 2.1. Now to find sufficient conditions for $\beta\left(2^{X}\right)=2^{\beta X}$ is to find conditions which imply that $2^{X}$ is $C^{*}$-embedded in $2^{\beta X}$. In this approach, we are asking when will $2^{\beta X}$ have the properties, as a compactification of $2^{X}$, that characterize $\beta\left(2^{X}\right)$ as a compactification of $2^{X}$ ? Without question, this is the most obvious and most direct means of approaching the condition $\beta\left(2^{X}\right)=2^{\beta X}$. However, the approach taken here is to reverse the roles of $\beta\left(2^{X}\right)$ and $2^{\beta X}$. We take the point of view that $2^{\beta X}$ has the nice properties, and that the relation $\beta\left(2^{X}\right)=2^{\beta X}$ says that certain structure on $X$ imposes these properties on $\beta\left(2^{X}\right)$. That is, we propose to describe $2^{\beta X}$ as a compact extension of $2^{X}$, and try 
to determine when $\beta\left(2^{X}\right)$ has the same description. The key to this approach is that $2^{X}$ and $2^{\beta X}$ are topological $V$-semilattices. $2^{\beta X}$ is a compact $V$-semilattice containing $2^{X}$ as a dense sub- $V$-semilattice, and continuous $V$-homomorphisms $2^{X} \rightarrow R$ extend continuously to $2^{\beta X}$. We thus describe $2^{\beta X}$ as a compact, algebraic extension of $2^{X}$, and determine conditions when $\beta\left(2^{X}\right)$ enjoys this algebraic structure.

We first recall the definition of a topological $V$-semilattice.

Definition 3.1. $A \bigvee$-semilattice is a set $Y$, equipped with a binary operation $\mathrm{V}$ that satisfies the following identities.

(i) $x \vee x=x$,

(ii) $x \vee y=y \vee x$,

(iii) $(x \vee y) \vee z=x \vee(y \vee z)$

If $Y$ is a $\vee$-semilattice and we define, for $a, b \in Y, a \leqslant b$ to mean that $a \bigvee$ $b=b$, then $\leqslant$ is a partial ordering on $Y$ relative to which every pair of elements has a least upper bound. Indeed, in this ordering, $\sup \{a, b\}=a \vee b$. Whenever we speak of order in a semilattice it is always of this natural order. We say a $V$-semilattice is $V$-complete, if every nonempty subset has a supremum. A subset $S$ of a $V$-semilattice $Y$ is called a sub-V-semilattice of $Y$ if $a, b \in S$ imply $a \vee b \in S$. A mapping $f: Y_{1} \rightarrow Y_{2}$ between $V$-semilattices $Y_{1}$ and $Y_{2}$ is a $\vee$-homomorphism if $f(a \vee b)=f(a) \vee f(b)$ for all $a, b \in Y_{1}$.

A topological $V$-semilattice is a $V$-semilattice $Y$ equipped with a topology relative to which the $V$-operation is continuous. When speaking of topological $V$-semilattices, terms like compact $V$-semilattice, continuous $V$-homomorphism, carry their obvious meaning.

Proposition 3.2. $2^{X}$ and $2^{\beta X}$ are topological $V$-semilattices. $2^{\beta X}$ is $a$ compact $V$-semilattice, and the natural mapping $i: 2^{X} \rightarrow 2^{\beta X}$ is a topological isomorphism of $2^{X}$ onto a dense $V$-complete sub- $V$-semilattice of $2^{\beta X}$. Every continuous $V$-homomorphism from $2^{X}$ to $R$ extends to a continuous $V$-homomorphism of $2^{\beta X}$ into $R$.

Proof. The first statement follows from statement (iv) in $\S 1$, the $V$ being the usual set-theoretic union. We know that the map $i$ is a homeomorphism, and clearly it preserves the operation. Therefore $i$ is a topological isomorphism of $2^{X}$ onto a dense sub- $V$-semilattice of $2^{\beta X}$. The natural ordering involved in these semilattices is just set-theoretic inclusion. Thus each semilattice is $V$-complete. Now $i\left(2^{X}\right)$ is a $\vee$-complete sub- $V$-semilattice of $2^{\beta X}$, since for $\left\{A_{j}: j \in I\right\} \subseteq$ $2^{X}, \bigvee_{j \in I} i\left(A_{j}\right)=\bigvee_{j \in I} \mathrm{cl}_{\beta X} A_{j}$ in $2^{\beta X}$ is just $\mathrm{cl}_{\beta X}\left(\bigcup_{j \in I} \mathrm{cl}_{\beta X} A_{j}\right)$ which equals $\mathrm{cl}_{\beta X}\left(\mathrm{cl}_{X}\left(\bigcup_{j \in I} A_{j}\right)\right)$ which lies in $i\left(2^{X}\right)$.

To prove the last assertion, let $g$ be a continuous $V$-homomorphism from 
$2^{X}$ into $R$. ( $R$, of course, carries the natural $\vee$ defined by $a \vee b=\max \{a, b\}$.) We claim that $g$ preserves all suprema. For, let $\left\{A_{i}: i \in I\right\} \subseteq 2^{X}$. We show that $g\left(\bigvee_{i \in I} a_{i}\right)=g\left(\mathrm{cl}_{X} \bigcup_{i \in I} A_{i}\right)$ coincides with $\bigvee_{i \in I} g\left(A_{i}\right)$. Clearly, since $g$ preserves order, $\bigvee_{i \in I} g\left(A_{i}\right) \leqslant g\left(\bigvee_{i \in I} A_{i}\right)$. For the sake of contradiction, assume $\bigvee_{i \in I} g\left(A_{i}\right)<g\left(\bigvee_{i \in I} A_{i}\right)$. By continuity, we can find a basic neighborhood $B\left(G_{0} ; G_{1}, \ldots, G_{n}\right)$ of $\bigvee_{i \in I} A_{i}=\mathrm{cl}_{X} \bigcup_{i \in I} A_{i}$ in $2^{X}$ such that $T \in$ $B\left(G_{0} ; G_{1}, \ldots, G_{n}\right) \Rightarrow g(T)>\bigvee_{i \in I} g\left(A_{i}\right)$. Now $\mathrm{cl}_{X} \bigcup_{i \in I} A_{i} \in$ $B\left(G_{0} ; G_{1}, \ldots, G_{n}\right)$ so we can find, for each $j=1,2, \ldots, n$, an index $i_{j} \in I$ so that $A_{i_{j}} \cap G_{j} \neq \varnothing$. Letting $P=\bigcup_{j=1}^{n} A_{i_{j}}$, we see that $P \in B\left(G_{0} ; G_{1}, \ldots, G_{n}\right)$, and thus that $g(P)>\bigvee_{i \in I} g\left(A_{i}\right)$. But $P=\bigcup_{j=1}^{n} A_{i_{j}}$, and $g$ preserves finite joins, so that

$$
g(P)=g\left(\bigvee_{j=1}^{n} A_{i_{j}}\right)=\bigvee_{j=1}^{n} g\left(A_{i_{j}}\right) \leqslant \bigvee_{i \in I} g\left(A_{i}\right)
$$

This is a contradiction. Thus $g$ preserves all joins, and this establishes our claim.

Now, let $f=g \mid\{\{x\}: x \in X\}$. Then $f \in C^{*}(X)$. We claim that $g=f^{s}$. But this now follows easily, since, for $T \in 2^{X}$,

$$
g(T)=g\left(\bigvee_{x \in T}\{x\}\right)=\bigvee_{x \in T} g(\{x\})=\bigvee_{x \in T} f(x)=f^{s}(T) .
$$

(We have thus shown that every continuous join homomorphism $2^{X} \rightarrow R$ has form $f^{s}$; it is easy to see that each $f^{s}$, for $f \in C^{*}(X)$, is a continuous $\vee$-homomorphism.) The continuous extension to $2^{\beta X}$ asserted in the proposition is simply $\left(f^{\beta}\right)^{s}$.

We thus see, by this proposition, that comparing $\beta\left(2^{X}\right)$ with $2^{\beta X}$ involves casting $\beta\left(2^{X}\right)$ in the role of an algebraic extension of $2^{X}$ with the properties in 3.2. We shall see, in the following theorem, that pseudocompactness enables us to impose the required algebraic structure on $\beta\left(2^{X}\right)$.

THEOREM 3.3. Let $2^{X} \times 2^{X}$ be pseudocompact. Then there is $a \mathrm{~V}$-semilattice structure on $\beta\left(2^{X}\right)$ relative to which $\beta\left(2^{X}\right)$ is a compact $V$-semilattice, and $2^{X}$ is a dense, sub-V-semilattice.

Proof. Let $u$ denote the continuous join operation on $2^{X}$. Being a continuous map from $2^{X} \times 2^{X}$ into $2^{X}, u$ has a Stone extension $u^{\beta}$ from $\beta\left(2^{X} \times 2^{X}\right)$ into $\beta\left(2^{X}\right)$. But $2^{X} \times 2^{X}$ is pseudocompact, so by Glicksberg's Theorem 1 of [5], $\beta\left(2^{X} \times 2^{X}\right)=\beta\left(2^{X}\right) \times \beta\left(2^{X}\right)$. Thus $u$ has a continuous extension $u^{\beta}: \beta\left(2^{X}\right) \times \beta\left(2^{X}\right) \rightarrow \beta\left(2^{X}\right)$. The map $u^{\beta}$ defines a continuous operation on $\beta\left(2^{X}\right)$. It is readily verified that $u^{\beta}$ defines a $V$-semilattice structure on $\beta\left(2^{X}\right)$. In order to check that the three identities in 3.1 hold for the operation $u^{\beta}$, one observes that, in each case, the identities are valid on a dense set, and since the identities are continuous functions of their variables, they hold everywhere. The 
remaining assertions in 3.3 are immediate. When the occasion arises, we will denote $u^{\beta}(s, t)$ by $s \vee t$, for $s, t \in \beta\left(2^{X}\right)$.

At this point we may pose the following question. Do 3.2 and 3.3 give enough information so that, with the assumptions in 3.3 , we are able to conclude that $\beta\left(2^{X}\right)$ and $2^{\beta X}$ are identical compactifications of $2^{X}$ ? This is indeed the case, but to prove it we will need several facts about topological $\vee$-semilattices. The first two facts are standard and easily proved results about compact $V$-semilattices, while the last two results are much more technical. We will content ourselves here with stating these results without proofs, giving appropriate references in each case.

3.4. Let $K$ be a compact $V$-semilattice. Let $\left(x_{\alpha}: \alpha \in D\right)$ be an increasing net in $K$. Then $\bigvee_{\alpha \in D} x_{\alpha}$ exists, and $\bigvee_{\alpha \in D} x_{\alpha}=\lim _{\alpha \in D} x_{\alpha}$. If $\left(x_{\alpha}: \alpha \in D\right)$ is a decreasing net bounded below, then $\bigwedge_{\alpha \in D} x_{\alpha}$ exists and $\bigwedge_{\alpha \in D} x_{\alpha}=$, $\lim _{\alpha \in D} x_{\alpha}$ (see [13], [16]).

3.5. A compact $V$-semilattice is $V$-complete ([13], [16]).

3.6. Let $f$ be a $\bigvee$-homomorphism from a compact $\bigvee$-semilattice $S$ onto a compact $V$-semilattice $T$. If $f$ preserves the suprema of increasing nets and the infima of decreasing nets, then $f$ is continuous [13].

3.7. Let $K$ be a compact $\bigvee$-semilattice. Then the continuous $\bigvee$-homomorphisms of $K$ onto metrizable compact $\bigvee$-semilattices separate the points of $K$. (See [7, p. 49].)

Now it is easy to see that, in $2^{X}$, the join of a subset $S \subseteq 2^{X}$ is the limit of the net of its finite subjoins, the net being directed by the finite subsets of $S$. This fact is tacitly proved in 3.2 in showing that continuous $V$-homomorphisms from $2^{X}$ into $R$ preserve suprema. From this fact, we see that $2^{X}$ is a $V$-complete subset of any compact $V$-semilattice $K$ in which $2^{X}$ is a sub-V-semilattice. Indeed, if $S \subseteq 2^{X}$, then, by the above remarks, the join of $S$ in $2^{X}$ is the limit of the increasing net of its finite subjoins. These finite subjoins in $2^{X}$ are the same as the corresponding joins in $K$. But, by 3.4, this net converges to the join of $S$ in $K$. Thus the join of $S$ in $K$ coincides with the join of $S$ in $2^{X}$. That is, $2^{X}$ is a $V$-complete subset of $K$. In particular, this statement is valid, in the case that $2^{X} \times 2^{X}$ is pseudocompact, for the compact $\bigvee$-semilattice $K=\beta\left(2^{X}\right)$.

We now have all the necessary tools to establish the main result of this paper.

THEOREM 3.8. Let $2^{X} \times 2^{X}$ be pseudocompact. Then $\beta\left(2^{X}\right)=2^{\beta X}$.

Proof. By statement (vi) in $\S 1, X$ considered as the singletons in $2^{X}$ is $C^{*}$-embedded in $2^{X}$. Thus the closure of this copy of $X$ in $\beta\left(2^{X}\right)$ is a copy of $\beta X$. So, there is a homeomorphism $h: \beta(X) \rightarrow \beta\left(2^{X}\right)$ such that $h(x)=\{x\}$ for each $x \in X$. Since $\beta\left(2^{X}\right)$ is the largest compactification of $2^{X}$, there is a quotient 
map $Q: \beta\left(2^{X}\right) \rightarrow 2^{\beta X}$, whose restriction to $2^{X}$ is the identity on $2^{X}$. We endow $\beta\left(2^{X}\right)$ with the $V$-semilattice structure described in 3.3. Since $Q$ is a $V$-homomorphism on a dense subset, by continuity, $Q$ is a $V$-homomorphism. Since a supremum is the limit of its finite subsuprema, by continuity, $Q$ preserves all suprema. Now, we define a mapping $F: 2^{\beta X} \rightarrow \beta\left(2^{X}\right)$ by $F(S)=\bigvee_{p \in S} h(p)$. Note that $F \mid \beta X=h$. We claim that $F$ preserves joins. For, let $\left\{A_{i}: i \in I\right\} \subseteq$ $2^{\beta X}$. Then

$$
\bigvee_{i \in I} F\left(A_{i}\right)=\bigvee_{p \in \cup_{i \in I} A_{i}} h(p)
$$

and

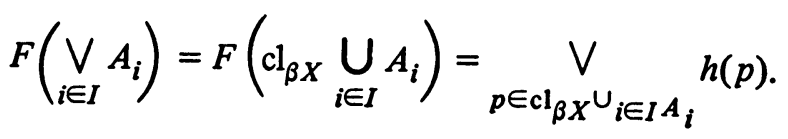

Clearly $\bigvee_{i \in I} F\left(A_{i}\right) \leqslant F\left(\bigvee_{i \in I} A_{i}\right)$. To prove the reverse inequality, let

$$
a=\bigvee_{i \in I} F\left(A_{i}\right)=\bigvee_{p \in \cup_{i \in I} A_{i}} h(p)
$$

Now the set $\left\{t \in \beta\left(2^{X}\right): t \leqslant a\right\}$ is clearly closed in $\beta\left(2^{X}\right)$. So, by the continuity of $h,\{p \in \beta(X): h(p) \leqslant a\}$ is closed in $\beta X$. Since this latter set evidently contains $\bigcup_{i \in I} A_{i}$, it therefore contains $\mathrm{cl}_{\beta X} \bigcup_{i \in I} A_{i}$. So, for all $p \in \operatorname{cl}_{\beta X} \bigcup_{i \in I} A_{i}$, $h(p) \leqslant a$. Thus

$$
F\left(\bigvee_{i \in I} A_{i}\right)=F\left(\mathrm{cl}_{\beta X} \bigcup_{i \in I} A_{i}\right)=\underset{p \in \mathrm{cl}_{\beta X} \cup_{i \in I} A_{i}}{\bigvee} \leqslant a=\bigvee_{i \in I} F\left(A_{i}\right)
$$

Combining the two inequalities, we conclude that $F$ preserves joins. Now consider the map $Q \circ F: 2^{\beta X} \rightarrow 2^{\beta X}$. By the continuity of $Q$ and $h, Q \circ F$ is the identity on $\beta X$ (that is, on the set $\{\{p\}: p \in \beta X\}$ ). Since $Q$ and $F$ preserve joins, so does $Q \circ F$. So if $S \in 2^{\beta X}$, we have

$$
Q \circ F(S)=Q \circ F\left(\bigvee_{p \in S}\{p\}\right)=\bigvee_{p \in S} Q \circ F(\{p\})=\bigvee_{p \in S}\{p\}=S .
$$

Thus, $Q \circ F$ is the identity on $2^{\beta X}$. It follows that $F$ is one-to-one. Let $L=$ $F\left(2^{\beta X}\right)$. Since $L$ contains the pseudocompact $2^{X}$ as a dense subset, $L$ is itself pseudocompact. Thus $F$ is an algebraic isomorphism of $2^{\beta X}$ onto the dense, pseudocompact sub-V-semilattice $L$ of $\beta\left(2^{X}\right)$. We now show that $F$ is continuous. Now, if the topological $V$-semilattice $S$ is a sub-V-semilattice of a compact $V$-semilattice, then a map $G$ into $S$ is continuous if, and only if, $R \circ G$ is continuous for all continuous join homomorphisms $R$ of $S$ into metrizable $V$-semilattices. Indeed, by 3.7, $S$ has the weak topology generated by such $R$. Thus, to show $F: 2^{\beta X}$ $\rightarrow L$ is continuous, we prove that if $R: L \rightarrow M$ is a continuous $V$-homomorphism 
of $L$ onto a metrizable $V$-semilattice $M$, then $R \circ F$ is continuous. But, for any such $R, M$ must be compact, since $M$, as a continuous image of $L$, is pseudocompact, and every pseudocompact metric space is compact. Thus $R \circ F$ is a map between compact $\vee$-semilattices. Since $R$ is a continuous $\vee$-homomorphism, $R$ preserves all joins. Since $F$ is an algebraic isomorphism, $F$ preserves all joins.

Therefore $R \circ F$ preserves all joins. The same type of argument shows that $R \circ F$ preserves decreasing meets. Appealing to 3.6, we see that $R \circ F$ is continuous. Thus $F$ is itself continuous. Thus $F\left(2^{\beta X}\right)$ is compact. Since the image of $F$ contains $2^{X}\left(2^{X}\right.$ is a $\vee$-complete subset of $\left.\beta\left(2^{X}\right)\right)$, we conclude that $F\left(2^{\beta X}\right)=$ $\beta\left(2^{X}\right)$. It follows that $F$ is a homeomorphism of $2^{\beta X}$ onto $\beta\left(2^{X}\right)$ fixing $2^{X}$ pointwise. Therefore $2^{\beta X}=\beta\left(2^{X}\right)$.

REMARK 3.9. It seems quite plausible that the pseudocompactness of $2^{X}$ is equivalent to that of $2^{X} \times 2^{X}$. This would establish the converse to 2.1 . The author has not been able to resolve this question, and leaves it open to the reader. Even if the exact converse of 2.1 holds, it is unsatisfying in a very significant way. It does not describe the relation $\beta\left(2^{X}\right)=2^{\beta X}$ in terms of properties of $X$. What is needed, of course, is a description of the pseudocompactness of $2^{X}$ in terms of $X$. Some progress on this question may be found in [4]

It is [8, Theorem 5] that for normal, $T_{1}$ spaces $X, 2^{X}$ is $\aleph_{0}$-bounded if, and only if, $X$ is $\aleph_{0}$-bounded. Since $\aleph_{0}$-boundedness is productive and implies pseudocompactness, we obtain the following corollary. $=2^{\beta X}$.

COROllary 3.10. Let $X$ be normal, $T_{1}$, and $\aleph_{0}$-bounded. Then $\beta\left(2^{X}\right)$

REMARK 3.11. The technique employed in 3.3 to impose algebraic structure of the Stone-Čech compactification can be applied to rather general situations. For example, if we are given a completely regular topological algebra $A$ of a given type, pseudocompactness can be used, just as in 3.3 , to obtain an algebraic structure on $\beta A$ of the same type, relative to which $\beta A$ is a compact topological algebra, and $A$ a dense subalgebra. This can be used to compare an algebraic compactification to the Stone-Čech compactification whenever the algebraic extension is uniquely determined. In particular, this method can be used to give a version of the Comfort-Ross Theorem 1.2 of [2]; for a totally bounded topological group $G$ and its Weil completion $\bar{G}, \beta G=\bar{G}$ if, and only if, $G$ is pseudocompact.

\section{REFERENCES}

1. R. L. Blair and A. W. Hager, Extensions of zero-sets and of real-valued functions, Math. Z. 136 (1974), 41-52.

2. W. W. Comfort and Kenneth A. Ross, Pseudocompactness and uniform comtinuity in topological groups, Pacific J. Math. 16 (1966), 483-496. 
3. Leonard Gillman and Meyer Jerison, Rings of continuous functions, University Ser. in Higher Math., Van Nostrand, Princeton, N.J., 1960. MR 22 \#6994.

4. J. Ginsburg, Some results on the countable compactness and pseudocompactness of $2^{X}$, Canad. J. Math. (to appear).

5. Irving Glicksberg, Stone-Čech compactifications of products, Trans. Amer. Math. Soc. 90 (1959), 369-382. MR 21 \#4405.

6. A. W. Hager, Some remarks on the tensor product of function rings, Math. Z. 92 (1966), 210-224. MR 33 \#1831.

7. K. H. Hofmann and P.S. Mostert, Elements of compact semigroups, Merrill, Columbus, Ohio, 1966. MR 35 \#285.

8. J. E. Keesling, Normality and properties related to compactness in hyperspaces, Proc. Amer. Math. Soc. 24 (1970), 760-766. MR 40 \#6507.

9. - Normality and compactness are equivalent in hyperspaces, Bull. Amer. Math. Soc. 76 (1970), 618-619. MR 40 \#8019.

10. On the equivalence of normality and compactness in hyperspaces, Pacific J. Math. 33 (1970), 657-667. MR 42 \#2418.

11. - Compactness related properties in hyperspaces, Lecture Notes in Math., vol. 171, Springer-Verlag, Berlin and New York, 1970.

12. K. Kuratowski, Topology. Vol. I, PWN, Warsaw; Academic Press, New York, 1966, pp. 160-187. MR 36 \%40.

13. J. D. Lawson, Intrinsic topologies in topological lattices and semi-lattices, Pacific J. Math. 44 (1973), 593-602. MR 47 \#6580.

14. Ernest Michael, Topologies on spaces of subsets, Trans. Amer. Math. Soc. 71 (1951), 152-182. MR 13, 54.

15. Kitti Morita, Completion of hyperspaces of compact subsets and topological completion of open-closed maps, General Topology and Appl. 4 (1974), 217-233.

16. D. P. Strauss, Topological lattices, Proc. London Math. Soc. (3) 18 (1968), 217-230. MR 37 \#3532.

17. P. Zenor, On the completeness of the space of compact subsets, Proc. Amer. Math. Soc. 26 (1970), 190-192. MR 41 \#6151.

DEPARTMENT OF MATHEMATICS, UNIVERSITY OF MANITOBA, WINNIPEG, MANITOBA R3T 2N2, CANADA

Current address: Department of Mathematics, University of Toronto, Toronto, Ontario, Canada M5S 1A1 